and $£ 5,505$, respectively). Regression analysis demonstrated that this increased spending for White British patients holds after accounting for age, gender, deprivation, LTC count, and primary care network $(\mathrm{p}=0.0002)$. Funnel plot analyses did not show significant patterns in GP practice-level variability in social care, emergency departments nor mental health service use. There was no clear individual-level relationship between primary care consumption and use of these other services.

Discussion These findings raise questions around ethnicitybased equity of care. Furthermore, they do not support the common narrative around differential access and gate-keeping at a GP practice-level affecting broader system costs. An increased focus on drivers of costs in social care rather than emergency or primary care settings may be needed for patients with SMI. However, the generalisability of these $\mathrm{B} \& \mathrm{D}$ results to the general population has not yet been explored.

\section{OP12 MORTALITY RISK FOLLOWING SELF-HARM IN YOUNG PEOPLE: AN EXPLORATION OF SELF-HARM AND SUICIDE USING THE NORTHERN IRELAND REGISTRY OF SELF-HARM}

${ }^{1} \mathrm{E}$ Ross*, 'D O'Reilly, ${ }^{2} \mathrm{D}$ O'Hagan, ${ }^{1} \mathrm{~A}$ Maguire. 'Centre for Public Health, Queen's University Belfast, Belfast, UK; ${ }^{2}$ Public Health Agency, Belfast, UK

\subsection{6/jech-2020-SSMabstracts. 12}

Background Suicide is the second leading cause of death in young people worldwide. Self-harm is a recognised predictor of future suicide and is most common in young people under the age of 24 years. The aim of this study was to estimate the risk of mortality following self-harm in adolescents, including death by suicide, and to examine the factors associated with this outcome.

Methods The Northern Ireland Registry of Self-Harm (NIRSH) collects information on all self-harm presentations to all Emergency Departments (ED) in NI. NIRSH data from 2012-2015 was linked to centralised electronic data relating to primary care registration, prescribed medication and death records. Logistic regression was employed to examine the factors associated with increased likelihood of self-harm and Cox regression to estimate mortality risk following self-harm and to examine the factors associated with the greatest risk of mortality.

Results The cohort consisted of all 395,771 individuals aged 10-24 years who were resident in NI on 1st April 2012 followed up until 31st December 2019. During the study 4,513 (1.14\%) young people presented with self-harm, $116(2.6 \%)$ of whom died during follow-up with 49\% $(n=57)$ of those deaths being by suicide. Rates of self-harm were highest in females, those aged 20-24 years (OR=3.47, 95\% CI 3.23$3.73)$, and those living in the most deprived areas $(\mathrm{OR}=3.10$, 95\% CI 2.80-3.42). Most individuals self-harmed via self-poisoning with psychotropic medications (68.6\%), followed by self-injury with a sharp object (24.1\%). Although only 57 of those who presented with self-harm went on to die by suicide they accounted for $28.8 \%$ of all deaths by suicide in this cohort. Those who presented with self-harm were 27 times more likely to die by suicide compared to those who did not present with self-harm after adjustment for age and sex $(\mathrm{HR}=27.20$, 95\% CI 19.86-37.25).
Conclusion This constitutes the first population-wide study of self-harm and suicide in young people in the UK and provides valuable information to inform suicide prevention strategies. Additional analysis is underway exploring variation in mortality risk based on ED care intervention and will be complete by the time of the conference.

The authors would like to acknowledge the help provided by the staff of the Honest Broker Service (HBS) within the Business Services Organisation Northern Ireland (BSO). The HBS is funded by the BSO and the Department of Health $(\mathrm{DoH})$. The authors alone are responsible for the interpretation of the data and any views or opinions presented are solely those of the author and do not necessarily represent those of the BSO.

\section{Wednesday 9 September}

\section{Smoking: Cessation Services to Policy}

\section{OP13 WE CAN QUIT2 - PRELIMINARY RESULTS OF A PILOT CLUSTER RANDOMISED CONTROLLED TRIAL OF A COMMUNITY-BASED INTERVENTION ON SMOKING CESSATION FOR WOMEN LIVING IN DISADVANTAGED AREAS OF IRELAND}

${ }^{1} \mathrm{CB}$ Hayes*${ }^{*}{ }^{2} \mathrm{~J}$ Patterson, ${ }^{1} \mathrm{E}$ Burke, ${ }^{1} \mathrm{~N}$ O'Connell, ${ }^{3} \mathrm{~F}$ Dobble, ${ }^{4} \mathrm{~J}$ Vance, ${ }^{1} \mathrm{C}$ Darker, ${ }^{5} \mathrm{D}$ Devane, ${ }^{3} \mathrm{~L}$ Bauld, ${ }^{3} \mathrm{~N}$ Dougall. ${ }^{1}$ Public Health and Primary Care, Institute of Population Health, Trinity College Dublin, Dublin, Ireland; ${ }^{2}$ School of Health and Social Care, Edinburgh Napier University, Edinburgh, UK; ${ }^{3}$ Usher Institute, College of Medicine and Veterinary Science, University of Edinburgh, Edinburgh, UK; ${ }^{4}$ Irish Cancer Society, Dublin, Ireland; ${ }^{5}$ HRB Trials Methodology Research Network, NUI Galway, Galway, Ireland

\subsection{6/jech-2020-SSMabstracts. 13}

Background Tobacco use is the leading cause of preventable death worldwide. In Ireland almost 6000 smokers dye each year from smoking-related diseases. 'We Can Quit2' (WCQ2 is a pilot pragmatic two-arm, parallel group, cluster randomised trial of a community-based peer-led smoking cessation intervention for women living in disadvantaged areas. The aim is to explore feasibility and acceptability of trial processes including recruitment and retention rates. A future trial will assess the effectiveness on short and medium-term cessation rates.

Methods Four matched pairs of districts (eight clusters) selected by area level of deprivation, geographical proximity, and eligibility for free medical services were randomised to receive either WCQ (behavioural support + access to Nicotine Replacement Therapy (NRT)) delivered over 12 weeks by trained Community Facilitators (CFs) or to a one-to-one smoking cessation service delivered by health professionals from Ireland's Health Service Executive (HSE). Recruitment target: 24-25 women per cluster (97 per arm; 194 in total) in four waves with consent obtained prior to randomisation. Primary outcome: achievement of recruitment target. Secondary outcomes included retention and data completion rates at 12 weeks(w) and 6 months(m) post-quit date and proportion continuously absent from smoking at $12 \mathrm{w}$ (primary outcome for a future DT) and at $6 \mathrm{~m}$, (self-report + biochemical validation). Acceptability of trial processes and intervention delivery was assessed by interview with participants and community facilitators. 
Results 125/208 registered women consented to participate $(60.1 \%), 64.4 \%$ of the recruitment target (125/194). Recruitment was an iterative process and the target number (48) was reached in wave 4. Dropout rates were high, 67/125 (53.6\%) at $12 \mathrm{w}$ and $59 / 125(47.2 \%)$ at $6 \mathrm{~m}$. While the study was not powered to test effectiveness, $26.6 \%$ of women randomised to the intervention group were non-smokers at 12 weeks versus $16.6 \%$ of controls. Higher quit rates were observed in the intervention group, $(41.7 \%$ intervention, $25.8 \%$ control) in those who attended at least one delivery session (89). Differences in quit rates were not sustained at 6/12. High acceptability of trial processes and intervention delivery were reported.

Conclusion Recruitment to a community based smoking cessation pilot RCT for disadvantaged women was challenging but feasible. Challenges to recruitment and lessons learned will be discussed. A positive direction of effect in favour of the intervention was noted. Measures to improve retention rates are needed in a future definitive intervention trial.

\section{OP14 THE IMPACT OF HEALTH SYSTEM SUPPORT AND MIGRATION RELATED RISK FACTORS ON DUAL AND POLY-TOBACCO USE AMONG MALE ADULTS IN 15 LOW AND MIDDLE-INCOME COUNTRIES - IMPLICATIONS FOR GLOBAL TOBACCO CONTROL}

THC Chen* ${ }^{*}$ CM Millett, FTP Filippidis. School of Public Health, Imperial College London, London, UK

\subsection{6/jech-2020-SSMabstracts. 14}

Background Concurrently using two (dual users) or more than two (poly tobacco users) tobacco products is common in lowand middle-income countries, especially among men, and has been linked to increased health risks and nicotine addiction compared to single tobacco product use. While substantial inequalities persists with high tobacco use prevalence among disadvantaged groups, very little is known about the impact of health care support and migration status on tobacco use patterns among these population. This study aims to explore socioeconomic, health care and migration related risk factors of ploy tobacco users among men in 15 low-and middleincome countries.

Methods Data from 15 countries around the world were obtained from the most recent wave of the Demographic and Health Survey (DHS), collected between 2015 and 2018. A total of 155,425 men aged 15-49 were surveyed. Outcomes included single, dual and poly use, examined by education, wealth, health system support and migration status. Migration defined as whether the respondent slept or reside away from home in the last 12 months. Multilevel models were used to estimate the effect of covariates on tobacco use with along with fixed and random parameters.

Results In all studied countries, prevalence of dual and polytobacco use was highest in respondents with low socioeconomic status (SES). SES gradients in dual and poly use by education $(\mathrm{RR}=0.64$; 95\% CI: $0.55-0.67$ for higher education vs illiterates) and wealth index $(\mathrm{RR}=0.15 ; 95 \% \mathrm{CI}$ : 0.11-0.20 for richest vs poorest) were observed. Furthermore, results showed higher risks of being a poly user among migrant men $(\mathrm{RR}=1.70 ; 95 \% \mathrm{CI}: 1.65-1.71)$ and $\mathrm{a}$ lower risk against dual use for those covered by health insurance $(\mathrm{RR}=0.82 ; 95 \% \mathrm{CI}$ : 0.79-0.85). However, dual and poly tobacco use were not associated with a country's purchasing power parity.

Conclusion Positive associations were found between dual and poly tobacco use among male respondents with lower SES and migration backgrounds, whereas respondents protected by health care system were associated negatively. This is the first study to examine the impact of health system support and migration status among men in low and middle-income countries. Findings imply that policy makers should consider targeting populations with migration background and those with reduced access to health care to tackle with smoking inequality in tobacco control science.

\section{OP15 EXPLORING THE IMPACT OF SMOKE-FREE LEGISLATION ON EXPOSURE TO SECOND-HAND SMOKE AMONG NON-SMOKING ADULTS IN ENGLAND}

C Mathew*, C Kypridemos. Department of Public Health and Policy, University of Liverpool, Liverpool, UK

\subsection{6/jech-2020-SSMabstracts. 15}

Background In the presence of mounting evidence on the adverse effects of second-hand smoke (SHS) to non-smokers, England implemented smoke-free legislation on 1 July 2007 that rendered all public enclosed spaces smoke-free. As England considers becoming smoke-free by 2030, an evaluation of the existing smoke-free policy in England utilizing recent data becomes paramount in informing policymakers who are debating an expansion of the legislation in England. This project sought to examine trends in exposure to SHS among adult non-smokers in England from 2003-2015 in order to determine whether the legislation produced changes in SHS exposure among non-smoking adults and whether the policy had a differential impact by sex and socioeconomic status (SES).

Methods This study was an interrupted time series analysis of data on self-reported exposure to SHS among adult non-smokers that were obtained from Health Survey England and spanned the period between 2003 and 2015. The study used regression methods to examine trends in not only exposure to SHS as a binary variable but also the number of hours of exposure to SHS both before and after the ban. The analysis was conducted for the general adult non-smoking population as well as by sex and SES, using the quintile groups of the index of multiple deprivation.

Results The odd of exposure to SHS was falling annually by 9.1\% (95\% CI: $6.7 \%$ to $11.5 \%$ ), in relative terms, before the implementation of the smoke-free policy. The odds dramatically reduced by $189.4 \%$ (95\% CI: $100.2 \%$ to $318.7 \%$ ) as a result of the policy, but slowly increased since then by $1.7 \%(95 \%$ CI: -3.0 to $6.2 \%$ ) annually. The modelled prevalence of SHS exposure, declined drastically by approximately 50\%, from $40.5 \%$ (95\% CI: $34.5 \%-46.9 \%$ ) to $19.1 \%$ (95\% CI: $11.2 \%$ $30.6 \%)$, in the immediate aftermath of the ban. Nevertheless, the number of hours of SHS exposure declined after the ban. The policy was most effective in men, and it reduces both absolute and relative sex inequalities. When socioeconomic inequalities were considered, the policy was more effective in the most deprived group, and reduced absolute socioeconomic inequality, however, absolute inequality was increased as a result of the policy. The analysis was performed in SPSS v24.

Conclusion While the population-wide decline in SHS exposure following the ban is a treatment to the success of smoke- 